\title{
NEURAL NETWORK BASED SIGNATURE VERIFICATION MODEL FOR BANK CHEQUES WITH THREE SPECIMEN SIGNATURES
}

\section{S. DHANDAPANI}

Research Scholar, Bharathidasan University, Trichy, Tamil Nadu, India

\begin{abstract}
Researches in offline signature verification use a large number of genuine and forged samples for the verification purpose, whereas real time banking system uses only a limited number of genuine samples. To go along with the reality, this research paper proposes a system to verify signatures on bank cheques, using only 3 signature samples. It does not require forged signatures by skillful forgers, as it may lead to more number of cheque frauds in future. The entire system modeled is divided into recognition and verification phase. Recognition phase is further subdivided into supervised and unsupervised methods. In the supervised recognition method, the account number of the customer is given as input and the corresponding signature is retrieved. But, in the unsupervised recognition method, the signature is directly given as input and the signature matching the features of the query signature are retrieved. So, the unsupervised input method has increased the system efficiency by reducing the processing time. Here, Feed forward Back propagation Neural Network is used for the classification purpose. So, the proposed verification model goes with the real time banking process for verification of signature on cheque and yields excellent results with an overall accuracy of $91.33 \%$. Thus, this paper has successfully overcome the existing hurdles in offline signature verification, like requiring more number of samples and involving forging experts. It has also improved the efficiency of the system by the use of unsupervised recognition and verification method.
\end{abstract}

KEYWORDS: Non-Intrusive Nature, Pressure, Angle of the Pen \& Signing Speed

Received: Jun 02, 2017; Accepted: Jul 12, 2017; Published: Aug 05, 2017; Paper Id.: IJCSEITRAUG201711

\section{INTRODUCTION}

The signature is the authentic and unique representation of a person's identity. It has been widely accepted by the people, because of its passive and non-intrusive nature [8]. But the threat is that, it can be forged. When forgery has already crept in, a signature is doubted. Signatures other than the customer and at times, even their own large intra class variations are considered as forged signatures by experts. Forgery can be classified as simple forgery, random forgery and skilled forgery. In simple forgery, the imposter knows only the name or he may have seen the signature somewhere, previously. Using any one of them, he tries to imitate the signature. In random forgery, the imposter does not know the name or the shape of the signature. In skilled forgery, the imposter has the sample signature handy and after several practices, he tries to forge the signature.

Signatures play a vital role in banking procedures. So, verification of signature becomes essential, because of the imposter's attempt to forge documents and cheques [12]. Mostly, banks do not show much importance to verify signatures, because they have to process an enormous number of the cheques, everyday [13] and therefore, only physical verification of the signatures by the bank cheque authorizer is done. So, signature verification becomes an important one. Signature verification can be of two schemes, based on the acquisition method. If the signature is 
captured by a writing e-pad and a stylus, it falls under online verification scheme. If the signature is scanned or photographed, it is called offline scheme. Online verification has a higher accuracy and verification rate when compared to offline verification. It is because of the dynamic features captured like pressure, angle of the pen, signing speed, time taken to complete the signature, number of pen's ups and downs, forward and backward stroke variations, which are very tedious to imitate, whereas, the offline signature verification scheme has only the scanned, digitized copy of the signature. Online signature templates that are stored can be hacked and the features can also be changed according to the imposter [11]. But, this is not the case in offline signatures. This adds merit to offline signature verification. So, offline signature's static characteristics can be extracted from the signature like geometric, texture features, etc. and used for verification process. Figure 1 shows the block diagram of the entire signature verification system.

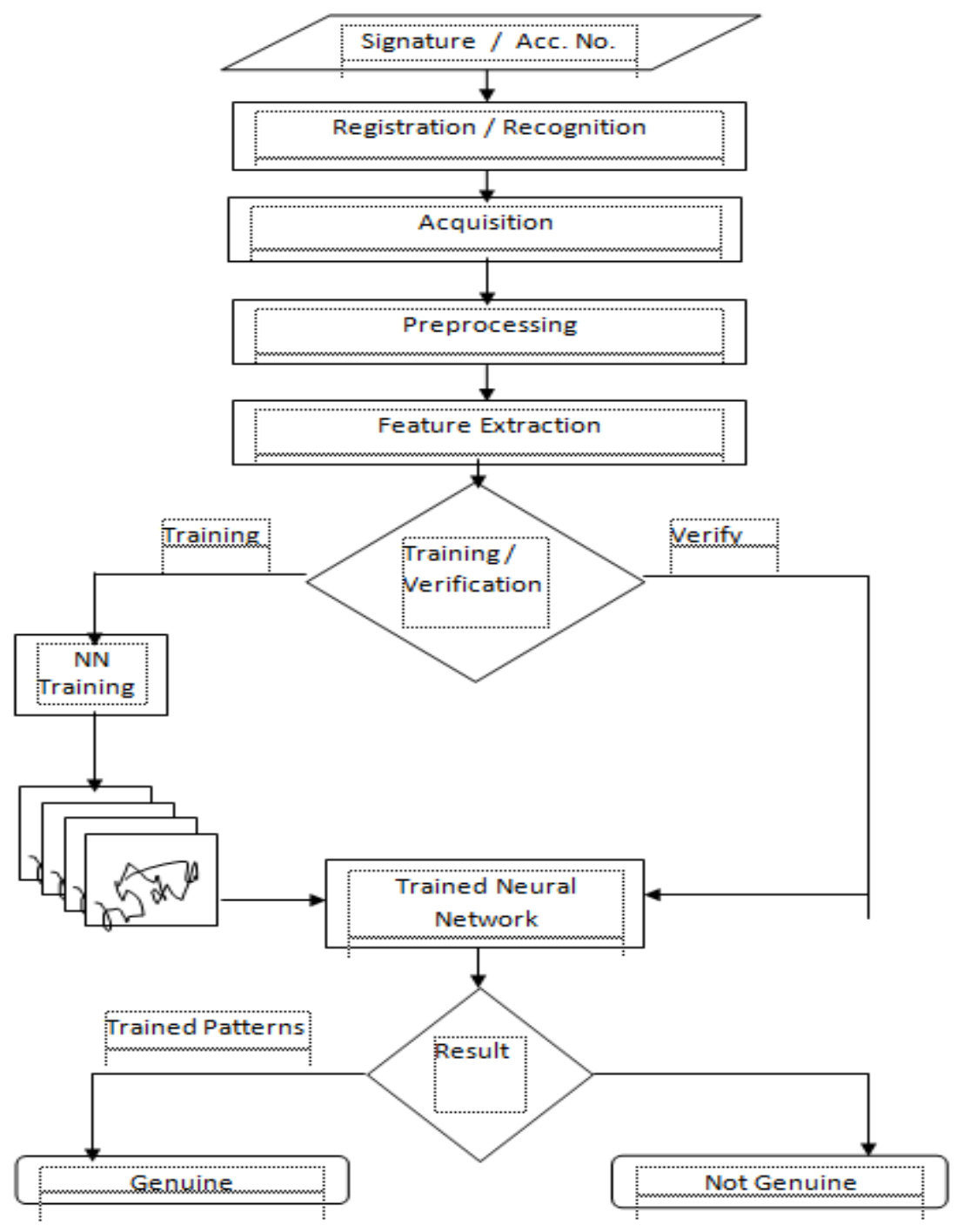

Figure 1: Proposed Signature Recognition and Verification System

The entire system, as shown in Figure 1, is divided into two modules, namely, recognition and verification modules. Recognition module is further subdivided into supervised and unsupervised modules. In supervised mode, to identify the signature, the account number is keyed and the corresponding signature to the account number is retrieved. But in an unsupervised mode, there is no need for any input, the signature alone can be given to the system. It matches with the 
existing system, and then, forwards the control to the verification module. Then, the feature set of the scanned signatures are extracted and used to train the feed forward back propagation neural network. Usually, when a cheque comes for collection, the bank officer keys in the account number of the cheque holder. The software gives the transaction history, balance amount in the account and the customer details. Since a handwritten signature is the vital key to pass a cheque for payment, a pop- up window with the specimen signature will be seen to aid the transaction. If there is an unusual pattern of transactions, new cheque bearer with higher amount and mutilated cheque, the officer verifies with all the specimen signatures collected from the customer for intra class variations. Still, if he has a doubt, he may call upon the customer to ensure that the cheque is issued with his knowledge and a genuine one. Now-a-days, signature verification is getting complex, even for forensic experts, who classify the signatures with an accuracy of 70\% [7]. So, the verification system modeled must be aware and cautious of the intra-class variations, non-availability of number of samples and rejecting a genuine signature [9].

The research article is organized in the following sections. Section II presents the literature review; Section III discusses the Acquisition and Preprocessing modules of the verification system. In section IV, the recognition module is discussed. Section V discusses the Verification module. Section VI presents the experimental results. Section VII discusses about the accuracy, performance, when number of samples are added to the dataset and final section concludes the research paper.

\section{LITERATURE REVIEW}

A plethora of research works is available for offline signature verification. Ashwin et al [1] has used pixel based comparison to identify signatures. If the $50 \%$ of the sample signatures pixels matched, they are considered as genuine. Jungpil [2] used the internal angles as features and neighborhood comparison to evaluate signatures. Mayank et al [3] used the multiclassifier combining both the static and dynamic features. Texture and topological features are extracted for offline features, and this hybrid combination yielded a high accuracy rate. Sangeetha and et al [4] uses shape context for signature verification on bank cheques. They used k-nearest neighborhood classifier and the system works better for signatures on complex background like bank cheques. Juan and Youbin [14] used texture features based on gray-level as pseudo-dynamic features which reflect the writing strength. Histogram of oriented gradients was also used as a pseudo dynamic feature and got good results. Ashok Kumar and Dhandapani [15] extracted GLCM features from the signature image to train a neural network and the accuracy attained was $92.08 \%$. Javier [6] proposed a complimentary approach to offline signature verification. They proposed wide baseline matching and evaluation by local interest point matching techniques. They used Bayes classifier and yielded an FRR of 16.4\% and FAR of 14.2\%. Ashok Kumar and Dhandapani [16] used connected components labeling technique to find the number of connected components and extracting properties of each blob and found that when using fractal dimensions as one of the feature, the accuracy rate increased. They used neural networks for training the feature set and the same authors [17] extracted features using the concentric circles masking method to extract features. Since the signatures are encircled by concentric circles from the centroid of the signature image, the extracted features remain rotation invariant. Different neural network training functions are used for the training purpose and found that CCMM features show higher accuracy for traincgf function with an accuracy of 97.89\%. Miguel and et al [18] blended the signatures from the popular database with cheques and invoices with complex background and found that basic version of local binary pattern are more robust than rotation invariant uniform local binary pattern. They used support vector machine with histogram oriented kernels for classification. 


\section{ACQUISITION AND PREPROCESSING}

In signature verification, the process of digitizing the cheque is called Acquisition. The signature on the bank cheque is acquired using a high resolution flat bed scanner or a fixed camera. To improve the quality of acquisition, flash light can be avoided while capturing in the camera. For better results, the camera with lightning setup and pre-focused on the scan bed with edge positioning fixed on the top and left corner to the cheque is chosen. The signature can be extracted from the scanned cheque by several means. Simplest means of extraction is the difference method. Each bank has its cheque designed to have a specific measurement, size, color, etc. The area to be signed is predefined according to Cheque Truncation System (CTS). Usually, the predefined area of the cheque is in the right bottom portion of the cheque. So, a blank cheque image can be subtracted from the signed check image to extract the signature alone.

Pre processing increases the system efficiency by processing the signature part alone, instead of the entire image. The signature part of the image is alone cropped and binarised [10] and then noise is cleaned. This minimizes the time required and improves the efficiency of the system. Figure 2. Shows the preprocessing step images i.e. a) shows the empty cheque b) shows the sample cheque with signature alone,

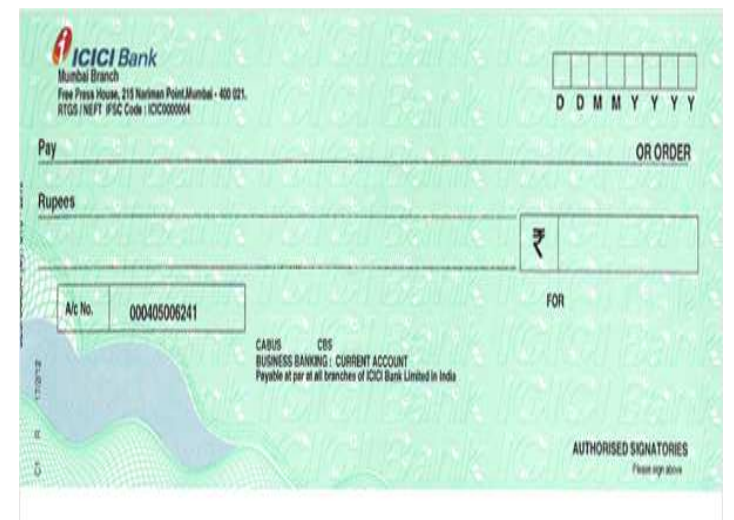

a) Empty Cheque Image

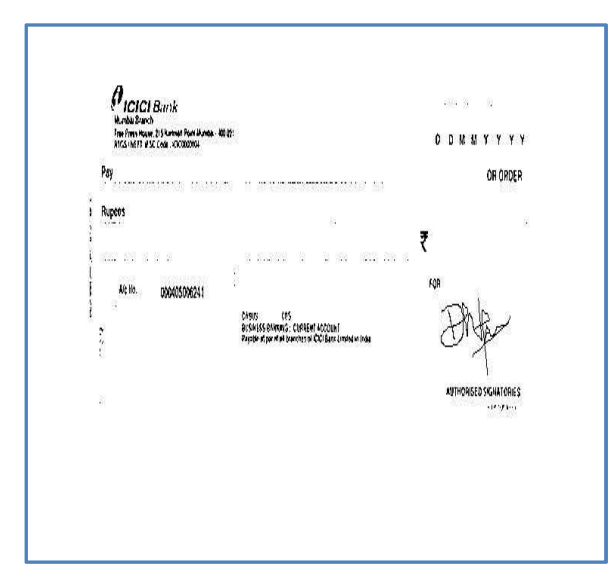

c) Binarised Cheque Image

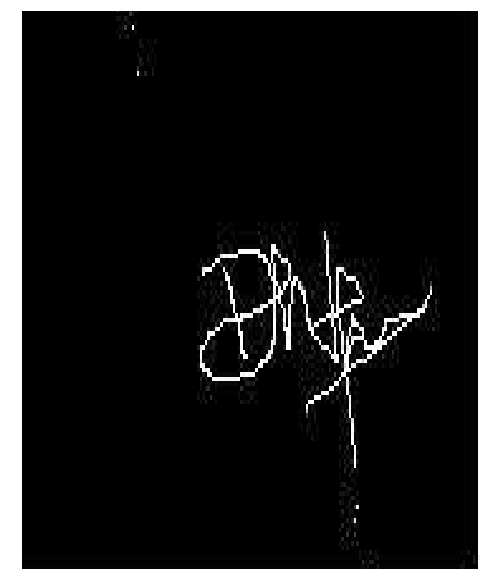

d) Signature Part Cropped
b)Signed Cheque

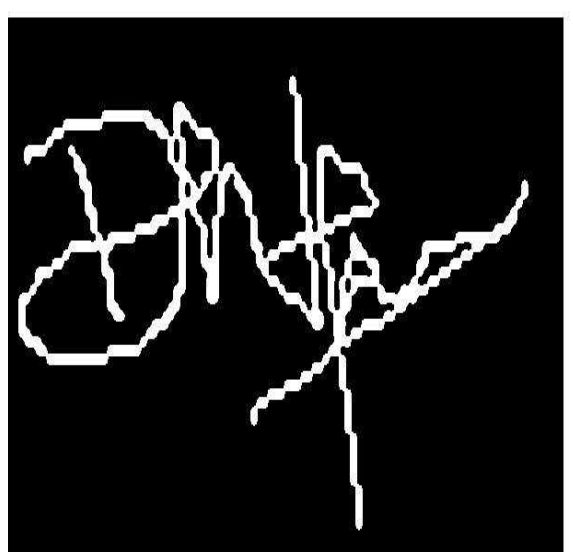

e) Enhanced Signature

Figure 2: Acquisition and Preprocessing of the Signature

Figure 2: c) shows the binarised image of the signed cheque, d) shows the cropped portion of the signature alone with some noise. And finally, e) shows the signature with the noise filtered and enhanced, which will be effective for feature extraction and further analysis. 


\section{SIGNATURE RECOGNITION}

Signature Recognition is the process of identifying the queried signature with the already existing signatures. In real time scenario, when a cheque comes for collection, the clearing officer in the bank enters the account number printed on the cheque to the system. Then, he checks the balance amount for the cheque clearance and compares the signature which is already scanned into the system with the signature on the cheque. If everything is fine, he clears the cheque. Here in this paper, the recognition is done in either supervised or unsupervised method. In supervised method, the account number is given as input and the corresponding account details are retrieved. In unsupervised method, the cheque containing the query signature is acquired preprocessed and the features are extracted. The extracted signature features are stored in a matrix form. Using Euclidean distance based method, the minimum distance between the query signature and the existing signatures feature vector is recognized.

\section{SIGNATURE VERIFICATION}

Signature verification is the core part of the modeling. After recognition, verification is the module, which classifies the signature into genuine or forged. This paper has made a novel difference among all other papers in three ways. First in signature verification, the number of genuine samples required is minimized and second there is no involvement of skillful forgers to create forged signatures. The non-genuine signatures are synthesized using the existing genuine signatures and their variations. Signature sample synthesis is the generation of synthetic signature from a user's signature samples [20]. The variations are amplified and forged signatures are manufactured for the verification purpose. Third, in unsupervised mode, the query signature is alone given as input for classification purpose. So, the efficiency of the verification is increased by reducing the processing time as in supervised method by giving account number.

The acquired signature is preprocessed and features like fractal dimension, aspect ratio, circularity, centroid, amplitude and phase angle of the zernike moments, perimeter, area, eccentricity, filled area, major axis length, minor axis length and the orientation of the signature are extracted from the signature. The query signature is compared with the sample signatures using neural network classifier. The neural network is trained with the sample signature features and used to classify the query signature as genuine or forged.

\section{Neural Network}

A feed forward back propagation neural network is used to classify the signatures. The choice of neural network lies in its ability to generalize, tolerate noise, graceful degradation and speed which are mostly required for verifying signatures on bank cheques [5]. The symmetry features extracted are used to train the neural network. The neural network uses $75 \%$ of the data for training, $15 \%$ of the data for validation and $15 \%$ of data for testing. Levenberg-Marquardt method of updating weight and bias values are used for training.

Algorithm I: Backpropagation Training algorithm

Input: Initialize the weights to random values, stopping condition

Output: Trained network which has the output value with minimum error difference between actual and targeted output

\section{Method}


Step 1: While stopping condition are false, repeat Steps 2-9

Step 2: For each training feature vector, repeat Steps 3-8

\section{Feed Forward Propagation}

Step 3: Every input neuron $\left(\mathrm{X}_{\mathrm{i}=1 . . \mathrm{n}}\right)$ receives input signal $\mathrm{x}_{\mathrm{i}}$ and broadcasts this to all units in the above layer (the hidden units).

Step 4: Each hidden neuron $\left(\mathrm{Z}_{\mathrm{j}=1 . . \mathrm{p}}\right)$ sums its weighted input signals

$z_{-} i n_{j}=v_{o j}+\sum_{i} x_{i} v_{i j}$

applies its activation function (Sigmoid) to compute its output signal,

$z=f\left(z_{-} i n_{j}\right)$

and sends this signal to all units in the layer above (output units).

Step 5: Each output unit $\left(Y_{k}, k=1, \ldots, m\right)$ sums its weighted input signals,

$y_{-} i n_{k}=w_{o k}+\sum_{j} z_{j} w_{k j}$

and applies its activation function(sigmoid) to compute its output signal, the output (activation) of $\mathrm{Y}_{\mathrm{k}}$ is denoted by $y_{k}$.

$y_{k}=f\left(y_{-} i n_{k}\right)$

\section{Back propagation of Error}

Step 6: Each output unit ( $\mathrm{Y}_{\mathrm{k}=1 . . \mathrm{m}}$ ) receives a target pattern corresponding to the input training pattern, computes its error information term,

$\delta_{k}=\left(t_{k}-y_{k}\right) f^{1}\left(y_{-} i n_{k}\right)$

calculates its weight correction term (used to update $\mathrm{w}_{\mathrm{jk}}$ later),

$\Delta w_{j k}=\alpha \delta_{k} z_{j}$

calculates its bias correction term (used to update $\mathrm{W}_{\mathrm{ok}}$ later),

$\Delta w_{o k}=\alpha \delta_{k}$

and sends $\delta_{\mathrm{k}}$ to units in the lower layer.

Step 7: Each hidden unit $\left(\mathrm{Z}_{\mathrm{j}=1 . . \mathrm{p}}\right)$ sums its delta inputs (from units in the layer above),

$\delta_{-} i n_{j}=\sum_{k=1}^{m} \delta_{k} w_{j k}$,

multiplies by the derivative of its activation function to calculate its error information term, 


$$
\delta_{j}=\delta_{-} i n_{j} f^{1}\left(z_{-} i n_{j}\right),
$$

Calculates its weight correction term (used to update $v_{o j}$ later),

$$
\Delta v_{i j}=\alpha \delta_{j} x_{i},
$$

and calculates its bias correction term (used to update $v_{o j}$ later),

$$
\Delta v_{o j}=\alpha \delta_{j}
$$

\section{Update Weights and Biases}

Step 8: Each output unit $\left(Y_{k=1 \ldots m}\right)$ updates its bias and weights $(j=0, ., p)$ :

$$
w_{j k}(\text { new })=w_{j k}(\text { old })+\Delta w_{j k},
$$

Each hidden unit $\left(Z_{j=1 . . p}\right)$ updates its bias and weights $(i=0, . ., n)$ :

$$
v_{i j}(\text { new })=v_{i j}(\text { old })+\Delta v_{i j} .
$$

Step 9: Test stopping condition.

Algorithm I give the basic back propagation algorithm. The input neuron receives the input and sums its weighted input signal to broadcast to the next layers by applying an activation function. Error is found by the difference between the expected and the target outputs at the last output layer. The weights and bias in the previous layers are adjusted depending upon the sum square error. The weight and bias correction terms are calculated and Back propagation takes place. For every iteration, the stopping criterion is checked. The iteration is stopped when the error reaches to a minimum value or 0 .

\section{Limitations of Offline Signature Verification}

In offline signatures, the process of signing is not known, so it makes signature verification more complex. In this process, high intra-personal variations are required for proper classification. But, more variations will lead to confusion between genuine and forgery. Complex genuine signature patterns varying at environmental, physical and mental conditions are also to be taken into account. An efficient and an ideal signature verification system pass all the above mentioned hurdles and emerge as a successful verification system that can be implemented in real time. In this paper, we have taken only three genuine signatures for verification. The average of the three is considered as the fourth signature sample. In order to classify signatures, a neural network needs the negative ones also. So the difference between the three signatures is found and multiplied twice and thrice of the mean signature to generate or synthesize signatures with large deviation from the original samples as shown in the table I.

Table 1 shows how to synthesize or produce the signature required for input to the Neural Network. First three columns contain the original signatures from which the remaining signatures are manufactured.

Table 1: Table Showing the Signature Synthesis Methodology

\begin{tabular}{|c|c|c|c|}
\hline Row No. & \multicolumn{1}{|c|}{ Calculation } & Aspect Ratio & Signature Type \\
\hline 1 & Sign. 1 & 4.547826 & \multirow{2}{*}{ Genuine Signatures } \\
\hline 2 & Sign. 2 & 3.596899 & \multirow{2}{*}{4.80198} \\
\hline 3 & Sign. 3 & & \\
\hline
\end{tabular}




\begin{tabular}{|c|c|c|c|}
\hline \multicolumn{4}{|c|}{ Table 1: Contd., } \\
\hline 4 & Avg. of above 3 ( row $1,2,3$ ) & 4.315569 & Mean Signature \\
\hline 5 & Avg. of 3 signs + Avg. of synthesized $*$ i(row $4+$ row 11$)$ & 5.922343 & \multirow{6}{*}{ Synthesized Signatures } \\
\hline 6 & Avg. of 3 signs - Avg. of synthesized $* \mathrm{i}($ row 4 and row 11$)$ & 2.708794 & \\
\hline 8 & Absolute(Sign.2-Sign.3) & 1.205081 & \\
\hline 9 & Absolute(Sign.1-Sign.3) & 0.254154 & \\
\hline 10 & Avg. of Absolute values & 0.803387 & \\
\hline 11 & $\operatorname{Avg}($ row 10$) * \mathrm{i}(\mathrm{i}=2$ to 16$)$ & 1.606775 & \\
\hline
\end{tabular}

The average of the 3 signatures can be taken as the fourth signature. The variations between the 3 genuine signatures are amplified to create the forged signatures and their features are extracted and fed to the Neural Network for classification purpose. Likewise 60 signatures are synthesized from the 3 genuine signatures.

\section{EXPERIMENTAL RESULTS}

The signature is acquired, preprocessed and features are extracted and are fed to the Neural Network for classification of signatures into genuine or forged. The experimental result values are tabulated in this section. The modeled system uses 10 sets of signature. Each set contains 3 genuine signatures. From this sample signatures, signatures are synthesized according to Table I. Totally we get 60 signatures of which 30 are genuine and 30 are non-genuine.

Table 2 shows the sample feature values for fractal dimension, aspect ratio, circularity, centroid, amplitude and phase angle of the zernike moments, perimeter, area, eccentricity, filled area, major axis length, minor axis length and the orientation of the signature.

Table 2: Extracted Feature Values of Sample Signatures

\begin{tabular}{|c|c|c|c|c|c|c|c|c|c|c|c|c|c|}
\hline FD & AR & Cirr & Centroid & Amp. & Ph. & Perime. & Area & Eccen. & FA & MAL & MiAL & Orienta. \\
\hline 1.335 & 5.089 & 0.000 & 265.220 & 62.143 & 0.028 & 176.211 & 403.806 & 1149.000 & 0.883 & 1688.000 & 104.927 & 49.207 & 53.365 \\
\hline 1.339 & 4.159 & 62.173 & 268.631 & 64.395 & 0.062 & 45.943 & 277.556 & 208.000 & 1.000 & 208.000 & 151.099 & 2.084 & 89.709 \\
\hline 1.338 & 5.990 & 8.144 & 275.566 & 53.180 & 0.098 & 143.613 & 490.676 & 1173.000 & 0.757 & 1175.000 & 87.710 & 57.312 & 57.843 \\
\hline 1.337 & 5.080 & 23.439 & 269.806 & 59.906 & 0.063 & 121.922 & 390.680 & 843.333 & 0.880 & 1023.667 & 114.579 & 36.201 & 7.166 \\
\hline 1.342 & 7.520 & 106.336 & 283.601 & 74.860 & 0.155 & 295.612 & 674.839 & 2130.000 & 1.204 & 2997.000 & 199.098 & 109.839 & 203.902 \\
\hline 1.344 & 8.740 & 147.784 & 290.498 & 82.337 & 0.201 & 382.457 & 816.919 & 2773.333 & 1.366 & 3983.667 & 241.357 & 146.658 & 302.270 \\
\hline 1.333 & 2.639 & 59.458 & 256.011 & 44.952 & 0.030 & -51.768 & 106.520 & 443.333 & 0.556 & 949.667 & 30.060 & 37.437 & 189.569 \\
\hline 1.331 & 1.419 & 100.906 & 249.113 & 37.475 & 0.076 & 138.613 & 35.560 & 1086.667 & 0.394 & 1936.333 & 12.199 & 74.256 & 287.937 \\
\hline
\end{tabular}

The Feed forward Back propagation Neural Network is used for verification. Neural Network is trained with the extracted feature values. Output values for the genuine signatures and synthesized signatures are tabulated in table III. The values close to 1 are genuine signatures and values close to 0 or not genuine signatures. The table 3 shows the output values for four sample signatures. In the first row of signature Sign.1, four genuine signatures show values close to 1 . So, they are all considered genuine signatures. In the second part, all the values show close to 0 , so all the 4 signatures of Sign.1 are not genuine signatures.

Table 3: Neural Network Output Values of Signatures

\begin{tabular}{|c|c|c|c|c|c|c|c|c|}
\hline & \multicolumn{4}{|c|}{ Genuine Signature NN Output Values } & \multicolumn{4}{|c|}{ Synthesized Signature NN Output Values } \\
\hline Sign. 1 & 1.002317 & 0.999744 & 0.939553 & 1.001467 & 0.093465 & 0.048104 & -0.03473 & -0.34724 \\
\hline Sign. 2 & 0.997145 & 0.980936 & 0.974027 & 1.00384 & 0.409707 & 0.004821 & 0.1933 & 0.048855 \\
\hline Sign. 3 & 0.753358 & 0.696519 & 0.876723 & 0.79202 & 0.137163 & -0.03245 & 0.071422 & 0.166403 \\
\hline Sign. 4 & 0.996463 & 0.987863 & 0.840204 & 0.995704 & 0.051692 & -0.02647 & 0.048698 & 0.000182 \\
\hline
\end{tabular}


Table 4 shows the verification results and the performance measure of the classification system modeled. It shows the true positive (TP) values, the number of correct positives, False Positive (FP), the number of incorrect positive values, True negative (TN), the number of correct negative values, false Negative (FN), the incorrect negative values, False acceptance Rate (FAR), the number of incorrect acceptance and False Rejection Rate, the number of incorrect rejections. Sensitivity is the measure of correct positives and specificity is a measure of correct negatives. Accuracy is the highest, when there are no incorrect positive or negatives.

Table 4: Performance Measures for 10 Signature Sets with Each 60 Signs

\begin{tabular}{|c|c|c|c|c|c|c|c|c|c|}
\hline Sign & TP & FP & TN & FN & FAR & FRR & SENSITIVITY & SPECIFICITY & ACCURACY \\
\hline 1 & 28 & 2 & 30 & 0 & 0.00 & 0.07 & 1.00 & 0.94 & 96.67 \\
\hline 2 & 23 & 7 & 17 & 13 & 0.43 & 0.23 & 0.64 & 0.71 & 66.67 \\
\hline 3 & 18 & 12 & 29 & 1 & 0.03 & 0.40 & 0.95 & 0.71 & 78.33 \\
\hline 4 & 30 & 0 & 30 & 0 & 0.00 & 0.00 & 1.00 & 1.00 & 100.00 \\
\hline 5 & 30 & 0 & 29 & 1 & 0.03 & 0.00 & 0.97 & 1.00 & 98.33 \\
\hline 6 & 30 & 0 & 30 & 0 & 0.00 & 0.00 & 1.00 & 1.00 & 100.00 \\
\hline 7 & 30 & 0 & 30 & 0 & 0.00 & 0.00 & 1.00 & 1.00 & 100.00 \\
\hline 8 & 25 & 5 & 30 & 0 & 0.00 & 0.17 & 1.00 & 0.86 & 91.67 \\
\hline 9 & 21 & 9 & 29 & 1 & 0.03 & 0.30 & 0.95 & 0.76 & 83.33 \\
\hline 10 & 29 & 1 & 30 & 0 & 0.00 & 0.03 & 1.00 & 0.97 & 98.33 \\
\hline
\end{tabular}

The average accuracy of the system is $91.33 \%$ for the 10 signature samples taken with only genuine signatures.

Table 5 shows the Receiver Operating Characteristic (ROC) plot values. There are three ROC curves for three different threshold values. Each ROC consists of the sensitivity and 1-specificity values for plotting, which is shown in Figure 3.

Table 5: 1-Specificity and Sensitivity Values for the ROC Plot

\begin{tabular}{|l|c|c|c|c|c|c|c|c|c|c|}
\hline & $\begin{array}{c}\text { Sign } \\
\text { Set 1 }\end{array}$ & $\begin{array}{c}\text { Sign } \\
\text { Set 2 }\end{array}$ & $\begin{array}{c}\text { Sign } \\
\text { Set 3 }\end{array}$ & $\begin{array}{c}\text { Sign } \\
\text { Set 4 }\end{array}$ & $\begin{array}{c}\text { Sign } \\
\text { Set 5 }\end{array}$ & $\begin{array}{c}\text { Sign } \\
\text { Set 6 }\end{array}$ & $\begin{array}{c}\text { Sign } \\
\text { Set 7 }\end{array}$ & $\begin{array}{c}\text { Sign } \\
\text { Set 8 }\end{array}$ & $\begin{array}{c}\text { Sign } \\
\text { Set 9 }\end{array}$ & $\begin{array}{c}\text { Sign } \\
\text { Set 10 }\end{array}$ \\
\hline 1-Specificity & 0.00 & 0.36 & 0.05 & 0.00 & 0.03 & 0.00 & 0.00 & 0.00 & 0.05 & 0.00 \\
\hline Sensitivity & 0.94 & 0.71 & 0.71 & 1.00 & 1.00 & 1.00 & 1.00 & 0.86 & 0.76 & 0.97 \\
\hline
\end{tabular}

Figure 3 shows the ROC curve plot. ROC curve is a performance measure of a classifier. It represents how FAR varies with respect to FRR. The true positive values with different thresholds are plotted against the false positives.

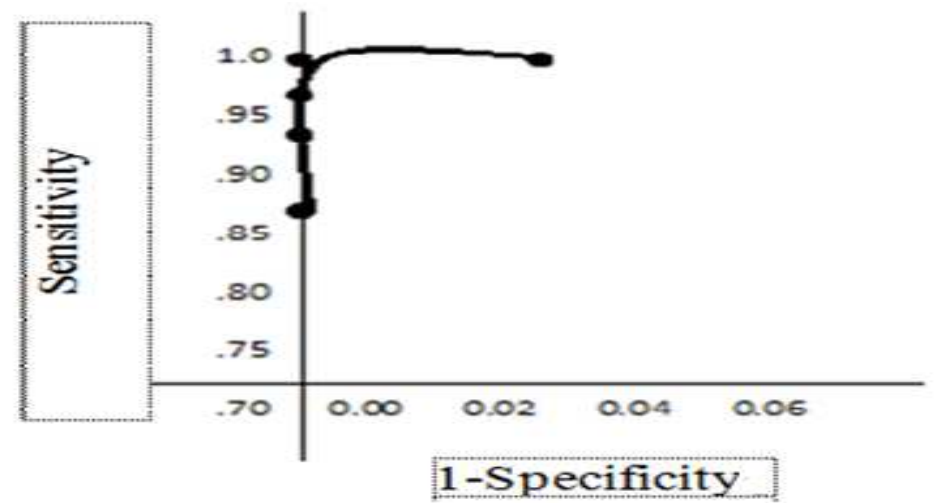

Figure 3: ROC

True positive is the sensitivity and the false positive is 1-specificity. When a diagonal line is drawn from the 
origin, the perfect classifier plot curve moves upward along the y axis towards the top left, and right curves at the end of the diagonal [19]. If the curve is above the diagonal, it is a good classifier and if the curve crosses the diagonal and comes below the diagonal, it is considered as a poor classifier or the classification accuracy is very low. The more the area of the curve above the diagonal, more the classification accuracy. The accuracy can be increased with increased number of signature samples from successful transactions.

\section{CONCLUSIONS}

Handwritten signature is a behavioral biometric, so, it changes according to the physical, mental and environmental conditions. No two signatures signed immediately are alike. If they are, they may be doubted. The main problem faced by offline signature verification is the number of signature samples. The proposed paper offers a solution, because, it does not require more number of genuine samples. It requires only 3 genuine samples. Moreover, it does not involve skillful forging experts to generate non-genuine signatures, which is always a great threat to the bank. The required signatures are synthesized with the existing signatures. Moreover, the system efficiency is increased by the unsupervised mode of recognition and verification of signatures. Usually, the account number is given to retrieve the account profile, but in this modeled system, the signature is directly given as input for recognition and verification. The system model has good classification accuracy of $91.33 \%$.

\section{REFERENCES}

1. Ashwin C.S, Harihar.V, Karthick.G, Karthik.A, Rangarajan K.R, PIXBAS-Pixel Based Offline Signature Verification, Advanced in Information Sciences and Service Sciences, doi: 10.4156/aiss.Vol 2(3), Sep, 2010.

2. Jungpil Shin and Weichen Lin, Shape Feature Extraction for On-line Signature Evaluation, eKNOW 2013 : The Fifth International Conference on Information, Process, and Knowledge Management, 2013.

3. Mayank Vatsa, Richa Singh, Pabitra Mitra, and Afzel Noore, Signature Verification Using Static and Dynamic Features, ICONIP 2004, Springer-Verlag Berlin Heidelberg LNCS 3316, pp. 350-355, 2004.

4. Sangeeta Girish Narkhede1, Prof. Dinesh D. Patil2, Signature Verification for Automated Cheque Authentication System Based on Shape Contexts, (IJCSIT) International Journal of Computer Science and Information Technologies, Vol. 5 (3), pp. $3297-3300,2014$.

5. Harpreet Anand and Prof. D.L Bhombe, Enhanced Signature Verification and Recognition Using Matlab, International Journal of Innovative Research in Advanced Engineering, Vol. 1(4), May 2014.

6. Javier Ruiz-del-Solar, Christ Devia, Patricio Loncomilla, and Felipe Concha, Offline Signature Verification Using Local Interest Points and Descriptors, CIARP 2008, Springer-Verlag Berlin Heidelberg, LNCS 5197, pp. 22-29, 2008.

7. Ravi Kumar, A.Sudhir Babu, Genuine and Forged Offline Signature Verification Using Back Propagation Neural Networks, International Journal of Computer Science and Information Technologies, Vol. 2 (4), pp. 1618-1624, 2011.

8. R. Plamondon and S. N. Srihari, Online and off-line handwriting recognition: a comprehensive survey, Pattern Analysis and Machine Intelligence, IEEE Transactions on, Vol. 22(1), pp. 63-84, 2000.

9. Luiz G. Hafemann, Robert Sabourin and Luiz S. Oliveira, Offline Handwritten Signature Verification - Literature Review, arXiv:1507.07909v2 [cs.CV] 19, August, 2015.

10. Sanjay N. Gunjal and Manoj Lipton, Robust Offline Signature Verification Based on Polygon Matching Technique, International Journal of Emerging Technology and Advanced Engineering, Vol. 1(1), November 2011. 
11. Iwasokun G. B., Udoh S. S and Akinyokun O. K, Multi-Modal Biometrics: Applications, Strategies and Operations, Global Journal of Computer Science and Technology: G Interdisciplinary Vol. 15(2), 2015.

12. Yazan M. Al-Omari, Siti Norul Huda Sheikh Abdullah, Khairuddin Omar, State-of-the-Art in Offline Signature Verification System, International Conference on Pattern Analysis and Intelligent Robotics, June 2011.

13. Jayadevan.R, Shaila Subbaraman and Pradeep M.Patil, Variance Based Extraction and Hidden Markov Model Based Verification of Signatures Present on Bank Cheques, International Conference on Computational Intelligence and Multimedia Applications, 2007.

14. Juan Hu and Youbin Chen, Offline Signature Verification Using Real Adaboost Classifier Combination of Pseudo-dynamic Features, $12^{\text {th }}$ International Conference on Document Analysis and Recognition, 2013.

15. Ashok Kumar. D and Dhandapani. S, A Bank Cheque Signature Verification System using FFBP Neural Network Architecture and Feature Extraction based on GLCM, International Journal of Emerging Trends \& Technology in Computer Science Vol. 3(3), May - June 2014.

16. Ashok Kumar. D and Dhandapani. S, A Novel Signature Verification System on Bank Cheque with Fractal Dimensions and Connected Components, International Journal of Applied Engineering Research, Vol. 10(14), pp. 34383-34389, 2015.

17. Ashok Kumar. D and Dhandapani. S, A Novel Bank Cheque Signature Verification Model using Concentric Circle Masking Features and its Performance Analysis over Various Neural Network Training Functions, Indian Journal of Science and Technology, Vol. 9(31), DOI: 10.17485/ijst/2016/v9i31/71863, August 2016.

18. Miguel A. Ferrer, J. Francisco Vargas, Aythami Morales, and Aaron Ordonez, Robustness of Offline Signature Verification Based on Gray Level Features, IEEE Transactions On Information Forensics And Security, Vol. 7, No. 3, June 2012.

19. Luiz S Oliveria and Edson Justino, Combining Classifiers in the ROC-space for Offline Signature Verification, JUCS, vol 14(2), pp. 237-251, Jan, 2008.

20. Liang Wan and Zhouchen Lin, Signature Sample Synthesis, Springer, DOI:10.1007/978-0-387-73003-5_7, pp. 1205-1210, 2009. 
